\title{
Über Pflanzen und Klima auf Coburg Island, N.W.T., Kanada
}

«Coburg sah, als Aufenthaltsort für Menschen betrachtet, nicht besonders einladend aus. Schwarze, senkrechte Bergwände fielen unmittelbar in die See. Alles, was Schlucht und Tal hieß, war voller Schnee und Eis. Kein einziges grünes Fleckchen konnten wir entdecken.» (SVERDRUP, 1903).

Die Pflanzendecke auf Coburg Island ist sehr karg. Von weitem scheint die rund $35 \mathrm{~km}$ lange und $10 \mathrm{~km}$ breite Insel tatsächlich wüst und leer, denn die wenigen Pflanzen verlieren sich in Sand und Geröll. In dieser polaren Wüste gedeihen nur 36 Arten von Blütenpflanzen. Vergleichsweise gibt es auf Axel Heiberg Island in der Umgebung des Basislagers $\left(79^{\circ} 25^{\prime} \mathrm{N} / 90^{\circ} 45^{\prime} \mathrm{W}\right.$ ) deren 127 (BESCHEL, 1963).

Die Feldarbeit wurde im Sommer 1976 im Rahmen des von Prof. F. Müller geleiteten «North Water Project» durchgeführt. Es war das Ziel, die Faktoren zu ergründen, welche die Dichte und Artenzusammensetzung der Vegetation auf Coburg Island bestimmen. Dabei sollte hauptsächlich die Wirkung von Klimafaktoren untersucht und soweit möglich der Einfluß einiger Bodenfaktoren abgeschätzt werden.

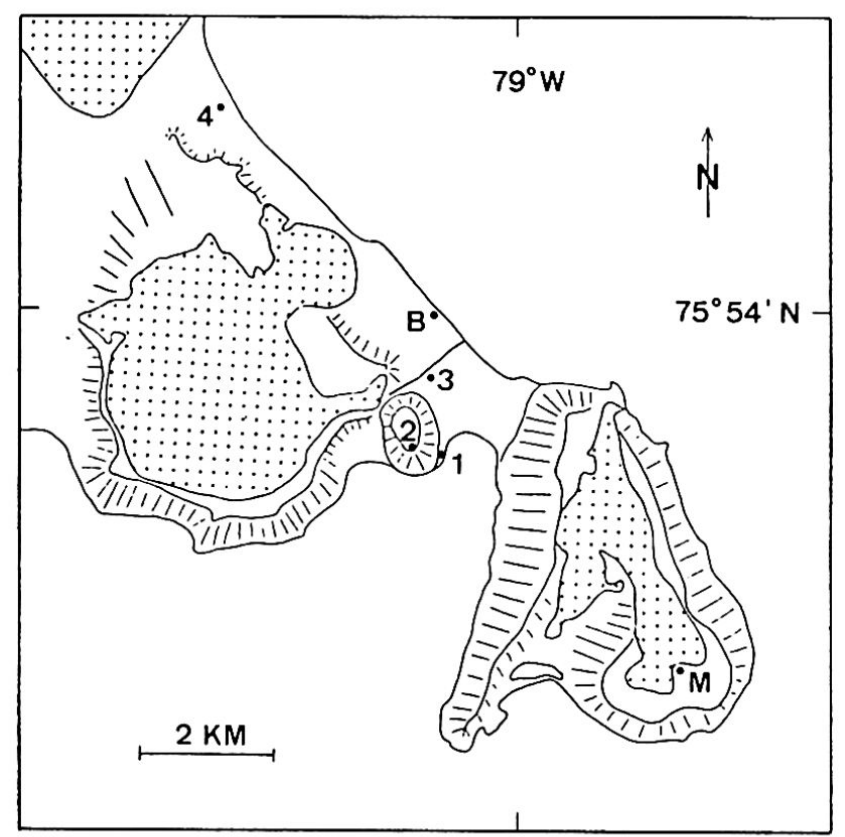

Fig. 1: Das Untersuchungsgebiet und die Lage der Wetterstationen. Gletscher sind punktiert dargestellt. Weitere Angaben im Text.

\section{Pflanzen}

Die Namen der gefundenen Arten von Blütenpflanzen sind in Tab. 1 zusammengestellt. Als Bestimmungsbuch diente die "Illustrated Flora...» von PORSILD (1964). Porsild's Angaben über die bevorzugten Standorte der einzelnen Arten gelten auch für die Pflanzen auf Coburg Island. Eine Ausnahme bildet Carex subspathacea. Sie wächst nicht in feuchtem Ton an geschützten Stränden, die bei Flut überschwemmt werden, sondern kommt ausschließlich auf trockenem Schotter, etwa 4-20 m über dem Meeresspiegel vor.

Tab. 1: Blütenpflanzen auf Coburg Island. $\mathrm{H}$ bedeutet die ungefähre Häufigkeit der Arten: 1 sehr selten, 2 selten, 3 vereinzelt, 4 zahlreich, 5 massenhaft.

\begin{tabular}{|c|c|}
\hline Nr. & Art \\
\hline 1 & Hierochloe alpina (Sw.) R. \& S. \\
\hline 2 & Alopecurus alpinus L. \\
\hline 3 & Phippsia algida (Sol.) R. Br. \\
\hline 4 & Poa arctica ssp. caespitans (Simm.) Nannf. \\
\hline 5 & Colpodium Vahlianum (Liebm.) Nevski \\
\hline 6 & Festuca brachyphylla Schultes \\
\hline 7 & Carex subspathacea Wormskj. \\
\hline 8 & Luzula confusa Lindeb. \\
\hline 9 & Salix arctica Pall. \\
\hline 10 & Koenigia islandica L. \\
\hline 11 & Oxyria digyna (L.) Hill \\
\hline 12 & Polygonum viviparum $\mathrm{L}$. \\
\hline 13 & Stellaria laeta Richards. \\
\hline 14 & Cerastium alpinum L. \\
\hline 15 & Arenaria rubella (Wahlenb.) Sm. \\
\hline 16 & Silene acaulis L. var. exscapa (All.) DC. \\
\hline 17 & $\begin{array}{l}\text { Melandrium apetalum (L.) Fenzl ssp. } \\
\text { arcticum (Fr.) Hult. }\end{array}$ \\
\hline 18 & Melandrium affine (J.Vahl) Hartm. \\
\hline 19 & Ranunculus nivalis L. \\
\hline 20 & Ranunculus Sabinei R. Br. \\
\hline 21 & Papaver radicatum Rottb. \\
\hline 22 & Cochlearia officinalis L. ssp. groenlandica (L.) \\
\hline 23 & Cardamine bellidifolia L. \\
\hline 24 & Draba Bellii Holm \\
\hline 25 & Draba nivalis Liljebl. \\
\hline 26 & Draba lactea Adams \\
\hline
\end{tabular}

Hans Müller, Geographisches Institut ETHZ, Sonneggstraße 5, 8006 Zürich. 
Nr. Art

27 Saxifraga caespitosa L. ssp. eucaespitosa Engl. \& Irmsch.

28 Saxifraga cernua $L$.

29 Saxifraga foliolosa $\mathrm{R}$. Br.

30 Saxifraga nivalis $\mathrm{L}$.

31 Saxifraga oppositifolia L.

32 Saxifraga rivularis L. s. lat.

33 Saxifraga tricuspidata Rottb.

34 Potentilla hyparctica Malte

35 Cassiope tetragona (L.) D.Don

36 Pedicularis hirsuta $\mathrm{L}$.

In der Umgebung des Basislagers lassen sich folgende Standorttypen unterscheiden:

A Das trockene Ödland liegt auf erhöhten Stellen innerhalb der von alten Strandlinien geprägten Ebene. Der sandige und steinige Boden ist äußerst trocken und i. a. schon früh schneefrei. Die Pflanzendecke ist sehr spärlich - oft sind Dutzende von Quadratmetern völlig kahl. Die wichtigsten Arten sind Nr. 8, 21, 31, 9, 14, 34 (Tab. 1). Auf diesem Standorttyp wurde am 14. Juni 1976 als erste blühende Pflanze ein Gegenblättriger Steinbrech (Nr. 31) gefunden.

B Das feuchte Ödland befindet sich ebenfalls in der Ebene, aber in Mulden, wo sich Wasser auf dem Permafrost anstaut. Die Vegetation ist auch hier nur karg. Die wichtigsten Arten sind Nr. 29, 8, 32, 23, 11, 3.

C Die Berge sind zu einem großen Teil von Geröllhalden bedeckt. Der trockene Boden besteht fast ausschließlich aus leicht rutschendem Geröll und Schutt. Coburg Island gehört zum präkambrischen kanadischen Schild und die meisten Gesteine sind sauer. Pflanzen kommen in den Geröllhalden nur vereinzelt vor. Die Artenzusammensetzung ist ähnlich wie bei A: Nr. 21, 8, 31, 27, 30.

D An Bachrändern ist der Boden sehr feucht. Die Pflanzen stehen zeitweise einige Zentimeter tief im strömenden Wasser. Die Vegetation ist ziemlich dicht und setzt sich hauptsächlich aus den folgenden Arten zusammen: Nr. 32, 28, 11, 23, 2, 4, 8, 19.

Innerhalb der von den vier Hauptgruppen eingenommenen Flächen gibt es inselartig Gebiete, wo die Pflanzendecke viel dichter und artenreicher ist. In den folgenden Kapiteln sollen die Gründe dafür gesucht werden.

Die Wachstumszeit dauerte 1976 von Mitte Juni bis Ende August (Fig. 2). An vielen Stellen begann sie viel später, denn am 22. Juni war die Ebene ums Basislager erst zur Hälfte schneefrei und in einzelnen Mulden blieb der Schnee gar bis gegen Ende Juli liegen. Es ist darum verständlich, daß auf Coburg Island nur eine einzige einjährige Art vorkommt, nämlich Koenigia islandica.

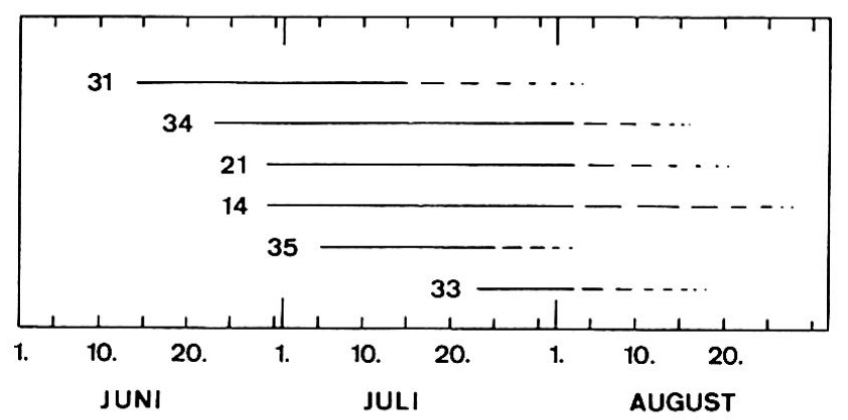

Fig. 2: Blütezeiten von ausgewählten Arten auf Coburg Island, 1976. Die Artennummern beziehen sich auf Tab. 1.

\section{Wetterstationen}

Als Teil des «North Water Project» bestanden bereits zwei Stationen (Fig. 1): Das Gebiet beim Basislager (B) auf $4 \mathrm{~m}$ ü. M. gehört zum Standorttyp A. Als Instrumente wurden u. a. ein Satz Thermometer, ein Anemograph und ein Schneemesser verwendet.

Marina Mountain (M) liegt auf $696 \mathrm{~m}$ ü. M. Der Boden ist mit groben Felsbrocken übersät und die Vegetation besteht nur aus Moosen und Flechten. Hier läuft eine automatische Wetterstation für Temperatur, Luftfeuchtigkeit und Wind. Im Sommer 1976 wurden vier zusätzliche Stationen aufgestellt:

Station 1 auf $21 \mathrm{~m}$ ü. M. stand in einer ungewöhnlich dichten und artenreichen Wiese. Als Instrumente dienten zwei Thermohygrographen in zwei Wetterhäuschen ( $20 \mathrm{~cm}$ und $150 \mathrm{~cm}$ über dem Boden), ein Anemograph und ein Regenmesser.

Station 2 auf 269 m ü. M. lag im Bereich des Standorttyps C. Es wurden dieselben Instrumente wie bei Station 1 verwendet. 
Die Umgebung von Station 3 auf $11 \mathrm{~m}$ ü. M. gehört zum Standorttyp D. Hier wurde ein Thermohygrograph in einem Wetterhäuschen in $20 \mathrm{~cm}$ Höhe über dem Boden eingesetzt.

Station 4 auf rund $15 \mathrm{~m}$ ü. M. war vom Standorttyp A umgeben. Auf diesem sehr steinigen Boden gedeihen äußerst wenige Pflanzen. Die Stelle ist extrem windexponiert und die Station wurde deshalb mit einem Anemographen bestückt.

\section{Temperatur}

Coburg Island grenzt ans North Water, d. h. an den Teil der nördlichen Baffin Bay, der selbst im Winter nie ganz von Eis bedeckt ist. Durch den mildernden Einfluß dieser offenen Meeresfläche bleibt es von September bis Mai deutlich wärmer als in den umliegenden Gebieten. Von Juni bis August andrerseits, d. h. während der Wachstumszeit, sind die Temperaturen ähnlich wie in der übrigen Arktis (MÜLLER et al., 1975). Für die Pflanzen sind aber ausschließlich die Sommertemperaturen von Bedeutung und sie können somit die mildernde Wirkung des North Water nicht nutzen. Wie sich aus Tab. 2 ersehen läßt, scheint es im Sommer bei der kontinentaler gelegenen Station auf Axel Heiberg Island sogar etwas wärmer zu sein.

Tab. 2: Durchschnittliche Monatsmitteltemperaturen in ${ }^{\circ} \mathrm{C}$

\begin{tabular}{|c|c|c|c|c|}
\hline $\begin{array}{l}\text { Coburg Island } \\
\left(74^{\circ} 54^{\prime} \mathrm{N} / 79^{\circ} 02^{\prime} \mathrm{W}\right)\end{array}$ & $\begin{array}{c}\text { Juni } \\
0,6\end{array}$ & Juli & August & Periode \\
\hline $\begin{array}{l}\text { Axel Heiberg Island } \\
\left(79^{\circ} 25^{\prime} \mathrm{N} / 90^{\circ} 45^{\prime} \mathrm{W}\right)\end{array}$ & 1,3 & 5,8 & 3,6 & $1960-76$ \\
\hline
\end{tabular}

Die Vegetationsunterschiede sind aber weit krasser, als es die Temperaturgegensätze erwarten lassen: Geschlossene Wiesen, wie sie auf Coburg Island einzig an sehr begünstigten Stellen vorkommen, sind auf Axel Heiberg Island nichts Ungewöhnliches.

Die lokalen Temperaturunterschiede auf Coburg Island werden zur Hauptsache durch die verschiedenen Eigenschaften von schneefreier Landoberfläche, Gletscher und Meer bestimmt. So war Ende Juni 1976 ein großer Teil der Insel schneefrei. Gleichzeitig war das Meer in der Bucht vor dem Basislager noch von Festeis, d. h. von einem an der Küste festgefrorenen Saum von Meereis bedeckt. An sonnigen Tagen stieg die Temperatur über der schneefreien Landoberfläche steil an, während die Luft über dem Eis deutlich kühler blieb. In der Grenzzone vermischten sich die beiden Luftmassen. Dabei wurde die Temperatur in der Küstenebene viel stärker beeinflußt als auf den nahen Bergen. Besonders im Juli kam es darum bei lange dauerndem Sonnenschein zu Inversionen, d. h. die Luft in den tiefer gelegenen Gebieten wurde so lange gekühlt, bis sie schließlich kälter war als auf den Bergen.

Am 25. Juli 1976 brach dann das Meereis vor dem Basislager auf und das offene Wasser - durch die Wellen in engem Kontakt mit der Luft - übernahm die abkühlende Wirkung des Eises. Die Temperaturabnahme mit der Höhe blieb deshalb weiterhin gering und betrug im Juli, dem eigentlichen Wachstumsmonat, zwischen den Stationen 1 und 2 im Durchschnitt nur $0,21^{\circ} \mathrm{C} / 100 \mathrm{~m}$. Dadurch wurden die Berg-Tal-Gegensätze verwischt und tatsächlich zeigt die Vegetation bis etwa $300 \mathrm{~m}$ fast keine Höhenabhängigkeit. Erst in noch höheren Lagen liegen die Temperaturen deutlich tiefer und sind beispielsweise auf Marina Mountain (696 m) so stark abgesunken, daß nur mehr einige Moose und Flechten gedeihen können.

Im August waren die mittleren Tagestemperaturen meist tiefer als die Temperatur des Meerwassers und dieses erwärmte deshalb die umgebende Luft. Dadurch verstärkten sich die Temperaturunterschiede zwischen Bergen und Gebieten in der Ebene. Die Luftschichtung zwischen den Stationen 1 und 2 war sogar oft absolut labil, so daß die warme, leichte Luft von der Bucht aufstieg und die kalte, schwere Luft vom Berg hinunterfloß. Der mittlere Temperaturgradient war darum im August mit $0,96^{\circ} \mathrm{C} / 100 \mathrm{~m}$ deutlich größer als im Juli. In der Ebene selbst waren die Temperaturunterschiede von Ort zu Ort sowohl im Juli als auch im August sehr gering. Die Pflanzenverteilung läßt sich damit keineswegs erklären.

\section{Wind}

Auf Coburg Island sind stark kanalisierte West- und Nordwestwinde in der Ebene sehr häufig (Fig. 3). Beim Basislager wurden Spitzengeschwindigkeiten von 
$90 \mathrm{~km} / \mathrm{h}$ gemessen und an besonders exponierten Stellen liegen die Werte noch deutlich höher. Es sind hauptsächlich solche Spitzenwerte, die eine große Wirkung entfalten. Einen guten Beweis dafür bilden schöne Windkanter, die verschiedentlich gefunden wurden. Auch der Einfluß auf die Vegetation ist bedeutend. Die Messungen zeigen, daß bereits schwache Winde verhindern, daß sich in Bodennähe eine deutlich wärmere, feuchtere und damit für die Pflanzen günstige Luftschicht ausbildet. Viele Arten sind an diese Bedingungen angepaßt, indem sie mit Behaarung und dichtem Wuchs in Polstern und Büscheln ermöglichen, daß um Stengel und Blätter windstille Lufthüllen entstehen. Verheerend sind erst stürmische Winde, deren erodierende Wirkung ein kleines Experiment aufzeigte: In der Ebene wurde ein $30 \mathrm{~cm}$ hoher Hügel aus der dort vorhandenen sandigen und trockenen Erde aufgetürmt. Bei Windgeschwindigkeiten von etwa $30 \mathrm{~km} / \mathrm{h}$ begannen einige Sandkörner zu rutschen. Besonders stark wirkten sich hohe Spitzenwerte aus und nach nur einem Monat waren $3 \mathrm{~cm}$ des Hügels abgetragen. Die heftigen Winde fegen also alle leichten Bestandteile weg, so auch abgestorbene Pflanzen. Das organische Material geht damit verloren

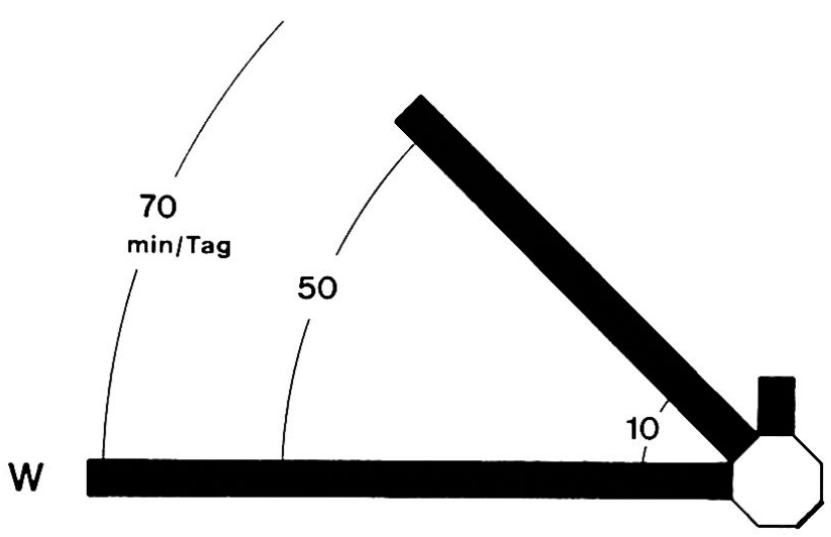

Fig. 3: Coburg Island, Basislager: Häufigkeit der Windrichtung im Sommer bei Geschwindigkeiten von $40 \mathrm{~km} / \mathrm{h}$ und mehr, umgerechnet auf einen "durchschnittlichen Tag».
Fig. 4: Grasbüschel und schwarze Flechten gedeihen im Windschatten von Steinen.

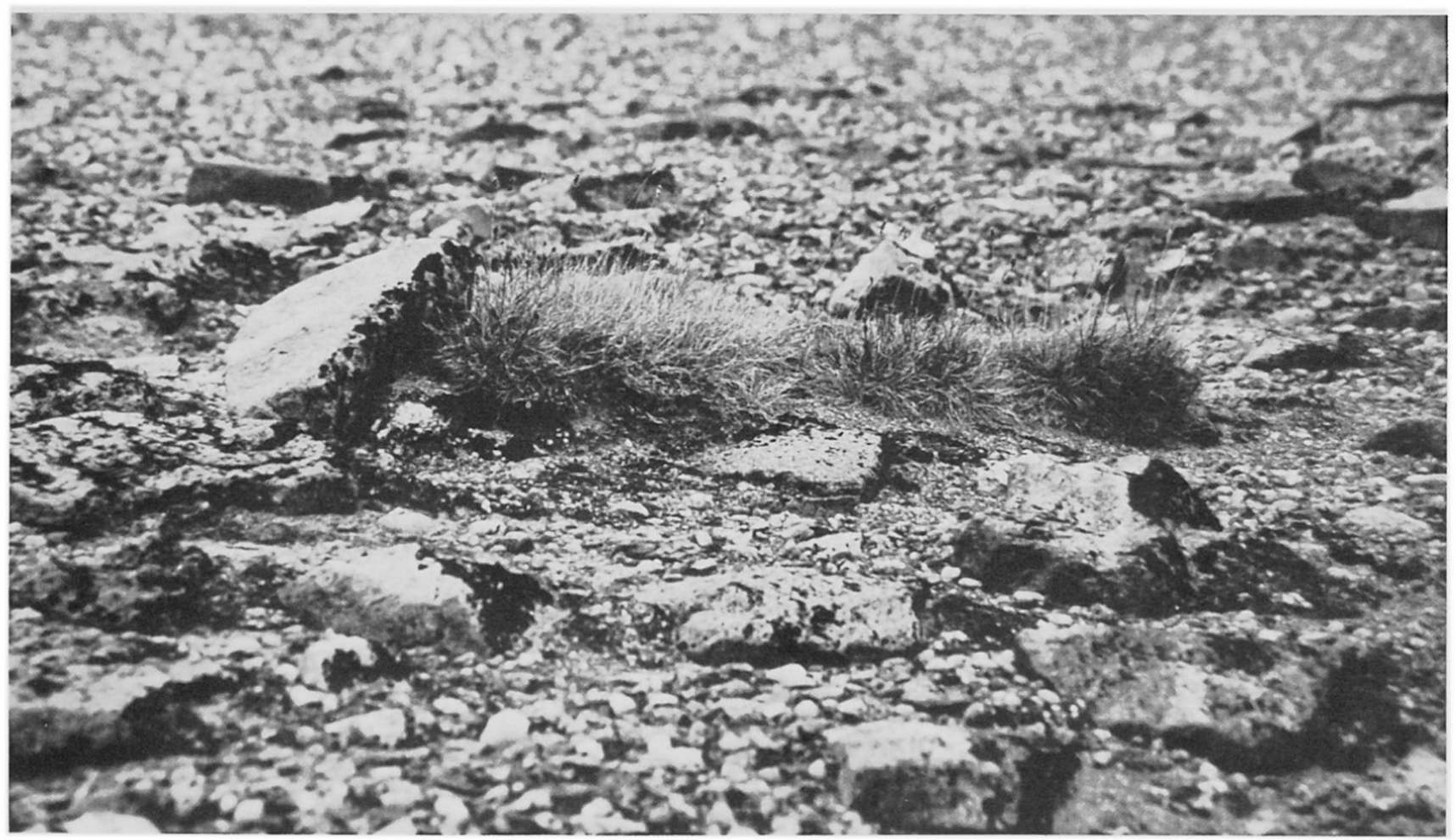


und zurück bleibt der für Coburg Island typische, humusarme und steinige Boden. Extreme Stürme entwurzeln sogar ganze Pflanzen.

Um vor solch zerstörerischen West- und Nordwestwinden Schutz zu finden, siedeln sich die Pflanzen vornehmlich in Mulden und hinter Steinen an. An exponierten Stellen in der Ebene zeigt sich das dadurch, daß alle Steine auf der windgeschützten ESESeite dicht von Flechten überwuchert sind, während die sturmgepeitschte WNW-Seite völlig kahl bleibt (Fig. 4). Aus dem gleichen Grund wachsen Moose oft wie Schneeverwehungen auf der ESE-Seite von Steinen. Fast alle größeren reichhaltigen Standorte sind vor West- und Nordwestwinden geschützt. Der Wind ist auf Coburg Island das deutlich wirkungsvollste Klimaelement.

\section{Stickstoff}

$\mathrm{Da} \beta$ es dem Boden an Stickstoff mangelt, ist daraus $\mathrm{zu}$ ersehen, daß die Vegetation um Knochenreste immer ungewöhnlich dicht ist. In der Ebene gedeihen mitten in absolut kahlem Geröll einige außerordentlich üppige, etwa kissengroße Gruppen von Luzula confusa, die sich als Nistplätze erwiesen. Die brütenden Vögel führen dem Boden mit ihren Exkrementen Stickstoff zu. Daraufhin wachsen die Pflanzen dichter, schützen sich so gegenseitig vor dem Wind und festigen mit den Wurzeln den sandigen Boden. Im nächsten Jahr werden erneut Vögel angelockt, womit sich der Kreis schließt und die Oase weiter wächst.

In der kurzen Zeit seit 1972 haben sich beim Basislager drei stark stickstoffliebende Arten angesiedelt, nämlich Cochlearia officinalis ssp. groenlandica, Phippsia algida und Saxifraga rivularis. Die letzteren zwei wachsen auf jenem unwirtlichen trockenen Boden, obwohl sie sonst feuchte Standorte vorziehen.

\section{Wasser}

Neben dem Wind scheint das Wasser der wichtigste Faktor zu sein, um die Pflanzenverteilung auf Coburg Island erklären zu können. Dichte und reichhaltige Vegetation gedeiht nur dort, wo der Boden genug Feuchtigkeit enthält, etwa entlang von Bächen und am Fuß von Bergen, wo sich Wasser auf dem Perma-

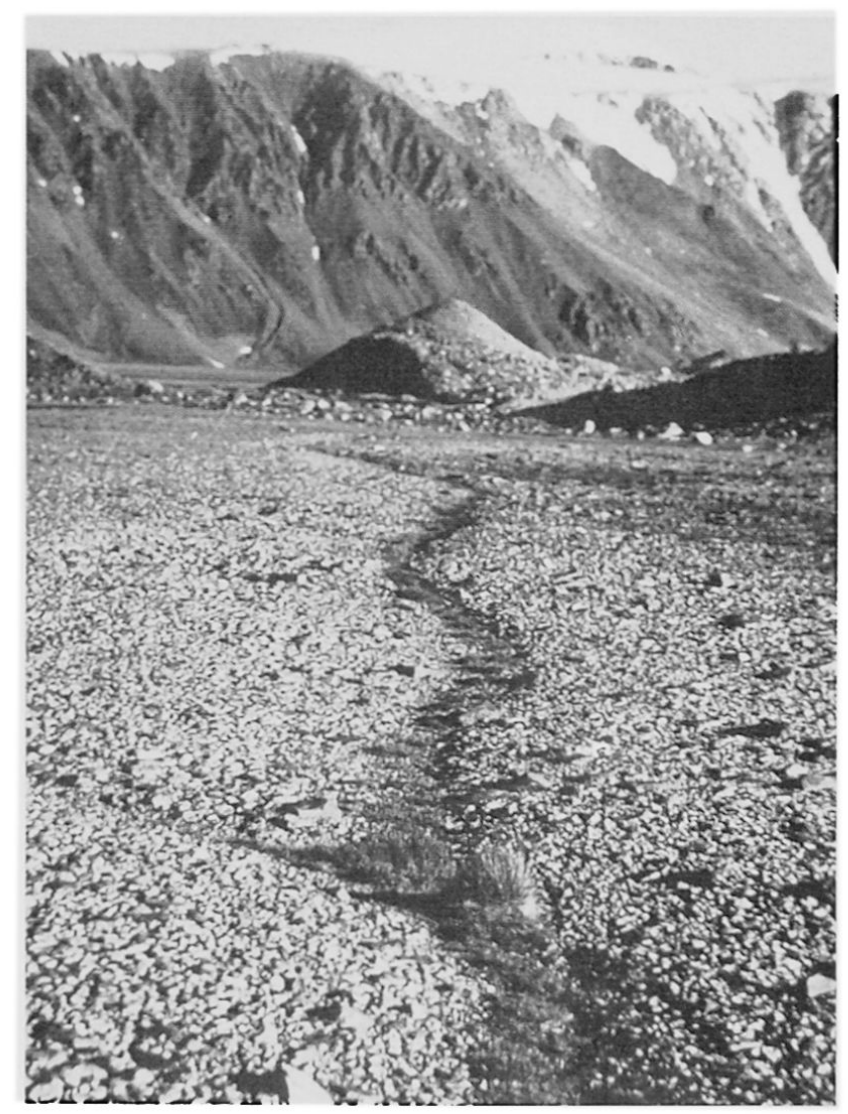

Fig. 5: Die Bodenfeuchtigkeit in den Eiskeilrinnen begünstigt den Pflanzenwuchs.

frost anstaut. Die Mächtigkeit der im Sommer 1976 aufgetauten Bodenschicht betrug zwischen 40 und $90 \mathrm{~cm}$.

In den etwa $20 \mathrm{~cm}$ breiten und $5 \mathrm{~cm}$ tiefen Eiskeilrinnen, die in der Ebene weit verbreitet sind, finden die Pflanzen ebenfalls etwas mehr Feuchtigkeit. Da sie darin zudem vor dem Wind ein wenig geschützt sind, gedeihen sie dort i. a. gut (Fig. 5).

\section{Ein junges Oekosystem}

In der Arktis standen den Pflanzen nach der letzten Eiszeit nur etwa 9000 Jahre zur Verfügung, um den nackten Fels zu besiedeln. Der Rückstand in der Entwicklung - etwa gegenüber den Tropen - dürfte eine nicht zu unterschätzende Ursache für die Kargheit der arktischen Vegetation sein (DUNBAR, 1968). Das Oeko- 
system auf Coburg Island ist noch jung. So gibt es beispielsweise keine klar umrissenen Artengruppen, die für gewisse Standorte kennzeichnend wären, denn die meisten Arten sind Pioniere, die kaum wählerisch sind, sondern überall vorkommen, wo die Lebensbedingungen nicht zu rauh sind. Seltenere Arten wachsen oft in Kolonien, d. h. sie kommen in einem kleinen Gebiet recht zahlreich vor, außerhalb aber, wo die Voraussetzungen genau gleich sind, fehlen sie völlig. Einzelne Pflanzengruppen sind also dabei, sich weiter auszubreiten.

Die Verbreitung der Arten über große Distanzen übernehmen in der Arktis hauptsächlich die Vögel und Winterstürme, welche die Samen auf dem glatten Meereis weit verfrachten können (SAVILE, 1972). Auf einer kleinen, stark vergletscherten und relativ isoliert liegenden Insel wie Coburg, wächst die Artenzahl so nur langsam. Mit großer Wahrscheinlichkeit ist die Besiedlung noch nicht abgeschlossen und die Dichte der Pflanzendecke dürfte im Laufe der Zeit stellenweise deutlich zunehmen.

\section{Verdankung}

Mein herzlicher Dank gehört in erster Linie Prof. Dr. F. Müller, A. Ohmura und G. Kappenberger, die mich in vielfacher Weise unterstützt haben. Die Untersuchung wurde durch das Canadian Department of En- vironment (Kontrakte OSX4-0098 und OSU76-00151) und den schweizerischen Nationalfonds (Kontrakt No. 2.855-0.73) finanziert. Wertvolle logistische Unterstützung gewährte das Polar Continental Shelf Project der kanadischen Regierung.

\section{Literatur}

BESCHEL, R. E. 1963. Geobotanical Studies on Axel Heiberg Island in 1962. Axel Heiberg Island, Preliminary Report 1961-1962, McGill University, Montreal, p. 199-215.

DUNBAR, M. J. 1968. Ecological Development in Polar Regions. A Study in Evolution. Prentice-Hall Inc., Englewood Cliffs, N. J.

MÜLLER, F. et al. 1975. The climate of North Water 1972-1975. North Water Project, Progress Report, ETH Zürich and McGill University Montreal, p. 14-54 (unveröffentlichter Bericht).

PORSILD, A. E. 1964. Illustrated Flora of the Canadian Arctic Archipelago. National Museum of Canada, Bulletin No. 146, 2nd edition, Ottawa.

SAVILE, D. B. O. 1972. Arctic Adaptations in Plants. Canada Department of Agriculture, Monograph No.6, Ottawa.

SVERDRUP, O. 1903. Neues Land - Vier Jahre in arktischen Gebieten. 1. Band, F. A. Brockhaus, Leipzig. 